\title{
Survival of APC-mutant colorectal cancer cells requires interaction between tankyrase and a thiol peroxidase, peroxiredoxin II
}

\author{
Dong Hoon Kang ${ }^{1,2}$, Joanna H.S. Lee ${ }^{1}$ E Sang Won Kang ${ }^{1,2, *}$ \\ ${ }^{1}$ Department of Life Sciences and ${ }^{2}$ Research Center for Cell Homeostasis, Ewha Womans University, Seoul 03760, Korea
}

\begin{abstract}
Overexpression of mammalian 2-Cys peroxiredoxin (Prx) enzymes is observed in most cancer tissues. Nevertheless, their specific roles in colorectal cancer (CRC) progression has yet to be fully elucidated. Here, a novel molecular mechanism by which PrxII/Tankyrase (TNKS) interaction mediates survival of adenomatous polyposis coli (APC)-mutant CRC cells was explored. In mice with an inactivating $A P C$ mutation, a model of spontaneous intestinal tumorigenesis, deletion of Prxll reduced intestinal adenomatous polyposis and thereby increased survival. In $A P C$-mutant human CRC cells, Prxll depletion hindered PARP-dependent Axin1 degradation through TNKS inactivation. $\mathrm{H}_{2} \mathrm{O}_{2}$-sensitive $\mathrm{Cys}$ residues in the zincbinding domain of TNKS1 was found to be crucial for PARsylation activity. Mechanistically, direct binding of Prxll to ARC4/5 domains of TNKS conferred vital redox protection against oxidative inactivation. As a proof-of-concept experiment, a chemical compound targeting Prxll inhibited the growth of tumors xenografted with $A P C$-mutation-positive CRC cells. Collectively, the results provide evidence revealing a novel redox mechanism for regulating TNKS activity such that physical interaction between Prxll and TNKS promoted survival of $A P C$-mutant colorectal cancer cells by Prxll-dependent antioxidant shielding. [BMB Reports: Perspective 2017; 50(8): 391-392]
\end{abstract}

*Corresponding author. E-mail: kangsw@ewha.ac.kr

https://doi.org/10.5483/BMBRep.2017.50.8.120

Received 7 June 2017

Keywords: Axin, Colorectal cancer, Peroxiredoxin, Tankyrase, $\beta$-catenin

Abbreviations: APC, Adenomatous polyposis coli; ARC, Ankyrin repeat cluster; Axin1, Axis inhibition protein 1; CK1, Casein kinase 1; CRC, Colorectal cancer; FAP, familial adenomatous polyposis; GSK, Glycogen synthase kinase; PARP; poly (ADP-ribose) polymerase; Prx, Peroxiredoxin; ROS, Reactive oxygen species; TNKS, Tankyrase

Perspective to: Dong Hoon Kang et al (2017) Nature Communications. Accepted online 2017. 05. 03, doi: 10.1038/s41467-01700054-0
Colorectal cancer (CRC) is a cause of the highest cancer mortality in developed countries and is characterized by inactivating mutations of the adenomatous polyposis coli (APC) suppressor gene. APC is a key scaffold protein in the $\beta$-catenin destruction complex, which is composed of the axis inhibition protein 1 (Axin1), $\beta$-catenin, casein kinase 1 (CK1) and glycogen synthase kinase (GSK)-3 $\beta$. Axis inhibition protein 1 (Axin1) tumor suppressor is another scaffold protein in the b-catenin destruction complex, but endogenous Axin1 proteins are tightly controlled by tankyrase-dependent degradation in colorectal cancer cells. However, the molecular mechanism regulating tankyrase (TNKS) activity in CRC remains largely unknown. To date, numerous studies have shown that initiation of intestinal tumorigenesis by APC mutations is promoted by the acquired or inherited mutation in the DNA glycosylase enzymes essential for base excision repair of oxidative DNA damage, suggesting the involvement of elevated levels of reactive oxygen species (ROS) in driving the intestinal tumorigenesis driven by APC mutations. Mammalian 2-Cys Prx enzymes are actually the most efficient peroxidases regulating cellular ROS level. These proteins catalyze the reduction of $\mathrm{H}_{2} \mathrm{O}_{2}$ to water in the presence of NADPH by coupling with the thioredoxin/thioredoxin reductase system. It has been well established that 2-Cys Prx enzymes have multifaceted roles in cellular ROS detoxification and signal transduction. This study primarily focused on elucidating a molecular mechanism involving TNKS and a thiol peroxidase named Prxll that may be necessary for the survival of CRC cells.

Somatic mutations on oncogenes and tumor suppressors cause intrinsic oxidative stress in cancer cells by amplifying ROS production. ROS has been recognized to serve a double-edged function, where a moderate and transient induction of cellular ROS levels is undoubtedly required for hyper-proliferation of cancer cells due to a second messenger role in growth factor signaling. In contrast, excessive production of ROS can be mutagenic and cytotoxic due to oxidative damage of macromolecules. Kang et al showed that loss of Prxll elevated the level of ROS and unexpectedly inhibited intestinal tumorigenesis induced by $A P C$ mutations, which further confirmed that $\mathrm{H}_{2} \mathrm{O}_{2}$ levels regulated by Prxll are 
critical for the survival of epithelial cancer cells in mouse intestinal adenomas. In CRC cells with an APC mutation, stringent knockdown of peroxidase activity of Prxll drastically reduced the levels of total and active (unphosphorylated) $\beta$-catenin via the canonical destruction complex. When $A P C$ is mutated, functional Axin1 becomes important for regulation of $\beta$-catenin levels. The level of Axin1 is known to be regulated by poly (ADP-ribose) polymerization (PARsylation) and sequential ubiquitination of the protein. Depletion of Prxll blocked PARsylation/ubiquitination of Axin1 without affecting total ubiquitination and caused the augmentation of functional Axin1-associated destruction complexes to degrade $\beta$-catenin, hence reversing the oncogenic phenotype conferred by the APC mutation. Taken together, these results suggested that Prxll-mediated regulation of $\mathrm{H}_{2} \mathrm{O}_{2}$ promotes epithelial cancer progression by targeting TNKS. This pathway does not require classical Wnt/B-catenin signalling, as APC mutations induce Wnt-independent accumulation of transcriptionally active $\beta$-catenin.

TNKS, the sole PARsylating enzyme for regulating the level of Axin proteins, specifically interacts with the glycine residue at position 116 of Prxll through its ankyrin repeat cluster (ARC) 4/5 domains. Endogenous Axin 1 proteins are tightly controlled by TNKS-dependent degradation via PARsylation and subsequent ubiquitination in CRC cells. It was shown for the first time that absence of Prxll reduced oncogenic $\beta$-catenin in the adenomatous polyps as well as the APC-mutant CRC cells due to the Axin1-dependent $\beta$-catenin degradation. It was also an intriguing result that $\mathrm{H}_{2} \mathrm{O}_{2}$-dependent inactivation of TNKS1 PARP activity happened as a result of the loss of zinc ions from the PARP domains. Taken together, the results reveal a novel redox mechanism by which a zinc-binding motif, essential for the PARP activity of TNKS, is vulnerable to oxidation and requires the Prxll- dependent antioxidant shielding effect (Diagram). Presently, there have been no isoform-specific chemical compounds that enable the inhibition of peroxidase activity of human Prxll. Conoidin A, a cell-permeable compound that covalently binds to parasitic Prxll, was tested and shown to be sufficiently inhibited by Prxl and Prxll activities using a slightly different mechanism. Interestingly, conoidin A inhibited colony-forming growth in APC-mutant CRC cells, but did not have the same effect on APC-competent CRC cells. Conoidin A treatment against tumor xenografts derived from APC-mutant CRC cells significantly retarded tumor growth. These results implied that a Prxll inhibitor may be used as a new therapeutic tool against with APC-mutant

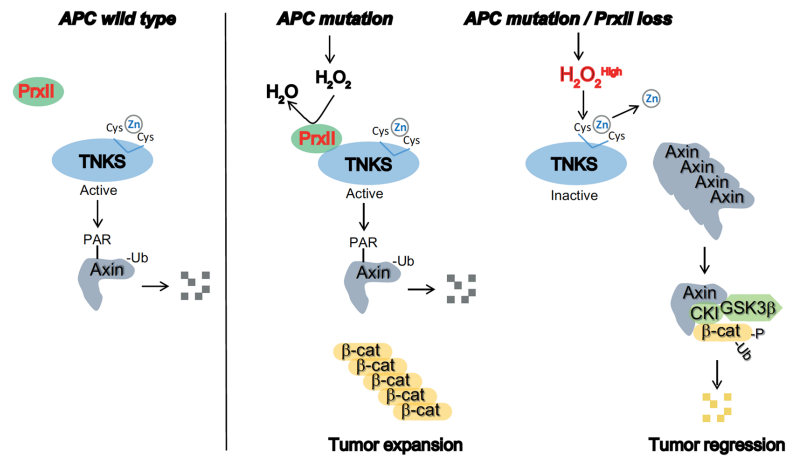

Diagram. Scheme for mechanistic model by which Prxll promotes survival of CRC cells. The level of intracellular $\mathrm{H}_{2} \mathrm{O}_{2}$ is elevated in the presence of an $A P C$ mutation, which in turn recruits and induces Prxll binding to TNKS. Prxll eliminates neighboring $\mathrm{H}_{2} \mathrm{O}_{2}$ molecules surrounding TNKS to shield the Cys residues in the zinc- binding motif upon oxidative stress, thus enabling the liberation of zinc ions from PARP domains. As a result, Prxll binding to TNKS confers a protective function against oxidative inactivation of TNKS PARP activity and consequently degrades Axin1. This promotes the accumulation of $\beta$-catenin, hence enforcing the proliferation and survival of CRC cells. Conversely, the loss or inhibition of Prxll in concert with an APC mutation prevents the reactions leading to the reduction of intracellular $\mathrm{H}_{2} \mathrm{O}_{2}$ levels, which then triggers Cys oxidation and leads to the release of zinc ions and inactivation of TNKS. Axin1 proteins accumulate soon after this process and contribute to the formation of the canonical $\beta$-catenin destruction complex. Destruction of $\beta$-catenins results in apoptotic cell death and regression of CRC.

CRCs.

In conclusion, the study provided intrinsic mechanistic evidence for a tumor-promoting role specific to Prxll, but not Prxl, beyond its canonical antioxidant role. Specifically, PrxII protected TNKS activity by directing binding to TNKS via ARC4/5 domains in the cytosol and consequently preserved the deregulated $\beta$-catenin pathway in APC-mutant CRC cells. Hence, targeting of Prxll may exert specific and broad therapeutic potentials for treating familial adenomatous polyposis (FAP) and $A P C$-mutant CRCs.

\section{ACKNOWLEDGEMENTS}

This work was supported by grants from the National Research Foundation of Korea (2014R1A2A1A01006934, 2012M3A9C 5048709 and 2012R1A5A1048236) and National R\&D Program for Cancer Control (1420280). 